\title{
A Fast and Updatable IP Address Lookup Scheme
}

\author{
Sang-Hun Chung, Sungkee Jean, Hyunsoo Yoon, and Jung-Wan Cho \\ Department of Electrical Engineering \& Computer Science \\ Division of Computer Science \\ Korea Advanced Institute of Science and Technology \\ \{shchung, skjean, hyoon, jwcho\} @ camars.kaist.ac.kr
}

\begin{abstract}
The Internet is growing very rapidly in both the size and the amount of traffic. This gowth has placed excessive strain on the Internet infrastructure, especially on routers. The IP address lookup is the operation that searches the longest matching prefix for the destination address of an incoming packet in order to determine the next hop of the packet. This operation is complex and is a major bottleneck in highperformance routers. In this paper, we propose a fast and updatable lookup scheme that can be easily implemented in hardware. We also present the memory allocation policy that supports for the incremental update of the forwarding table. Since our lookup scheme can be implemented with the small-bit logic and SRAM, the average delay per one lookup is about $18 \mathrm{~ns}$. That is, our scheme can achieve $55.56 \times 10^{6}$ routing lookups/s in average.
\end{abstract}

\section{Introduction}

The wide spread of the World Wide Web and the multimedia application make the Internet tremendous in both the size and the amount of traffic. The investigation [1] shows that the number of hosts on the Internet has been tripled within recent two years. Also, the traffic is doubling every few months [10]. This gowth has placed excessive strain on the Internet infrastructure, especially on routers. To solve the bottleneck of the Internet performance, faster links and high performance routers are required. The advance of fiber-optic transmission technology can support the bandwidth required in transmission medium. Therefore, routers are the key of the Internet performance. The IP address lookup is the single most time-consuming operation and typically defines the upper bound on the router's forwarding rate. Assuming that the average size of packet is

\footnotetext{
${ }^{0}$ This work was supported by the Korea Science and Engineering Foundation(KOSEF) through the Advanced Information Technology Research Center(AITrc)
}

$1000 \mathrm{bit}, 1 \mathrm{~Gb} / \mathrm{s}$ router must route a million packets per second. Hence, one lookup must have been completed within 1 $\mu \mathrm{s}$. Moreover, the IP address lookup is complex by the fact that entries in the forwarding table have variable lengths, and also that many entries may represent valid routes to the same destination. Thus, the IP address lookup has grown even more challenging in recent years. In this paper, we propose the fast and easily updatable IP address lookup scheme

Fundamental tasks of the router are the routing process and the packet forwarding process. The routing process deals with the network management such as running the routing protocols, creating and maintaining the forwarding table. The packet forwarding process determines the next hop (the output interface) of the packet based on information contained in the forwarding table, and copies the packet from the input interface to the proper output interface. The forwarding table search, called IP address lookup, is to find the longest matching prefix in the forwarding table. Since the advent of Classless Inter-Domain Routing (CIDR) [4], the forwarding table contains IP routes that consist of < prefix, prefix length $>$ pair, where prefix length is between 0 and 32. Due to CIDR, the traditional network class is obsolete and the exhaustion rate of IPv4 address space is slowed down. The example of the forwarding table and IP address lookup is as follows. If the forwarding table contains 143.248/16, 143.248.174/24, and 143.255/16 and the destination address of a packet is 143.248 .174 .50 , prefix 143.248 and prefix 143.248.174 match the destination address. Between these prefixes, 143.248.174 is the longest matching prefix.

Several hardware-implementable lookup schemes have been recently proposed $[3,5,6,8]$. Athough they have some advantages in aspect of the forwarding rate and the implemetation cost, almost of them did not support the incremental update in the forwarding table except for [5]. These schemes $[3,6,8]$ reduced the size of forwarding table significantly, but the internal fragmentation of the memory in update required either the whole table rebuilding or the dual memory. They did not consider the logic delay in hardware 
implementation and only emphasize on the number of memory accesses. In our scheme, we consider not only the size of forwarding table but also the incremental update of the forwarding table and the logic delay in lookup procedure.

The rest of this paper is orgnized as follows. Section 2 describes previous hardware-implementable lookup schemes. Section 3 describes our lookup scheme. Section 4 describes the performance analysis considering the logic delay. Section 5 states our conclusion.

\section{Previous Work}

We describe previous hardware-implementable schemes for IP lookup in aspect of the size of the forwarding table, the number of memory accesses and the incremental update of the forwarding table. The memory, typically SRAM, may be a dominant factor in implementation cost. The memory reference is a most time-consumming factor in lookup procedure. The update of the forwarding table may degrade the forwarding rate.

Degermark [3] proposed a compact forwarding table structure for fast lookup using L2 cache. This scheme can be implementable in both hardware and software. The size of the forwarding table which contains 40,000 prefix entries is about $150 \mathrm{~KB}$ and small enough to fit in L2 cache (SRAM in hardware implementation). The number of memory accesses is between 3 and 9 . The incremental update is not easy.

Gupta [5] developed a novel lookup scheme through the introducion of large memories(DRAM). This scheme utilizes the distribution of the prefix length, in which there are very few prefixes longer than 24-bits. The size of the forwarding table is fixed to $33 \mathrm{MB}$. The number of the memory access is between 1 and 2 . Since large memories made the update expensive, several update schemes also were proposed.

Huang [6] reduced the size of the forwarding table by utilizing the distribution of the prefix in a segment, which is based on the indirect lookup mechanism with variable offset length. By employing a modified form of Degermark's compression scheme, the table size can be further reduced. The size of the forwarding table is about $450 \mathrm{~KB}$ and the number of the memory access is between 1 and 3 . The incremental update is not easy by fact that the segment requires the variable sized memory and the internal fragmentation in memory occurs at the prefix insertion and deletion. Since prefix insertions and deletions may occur every few seconds, the performance might be degraded severely due to the memory bandwidth contention.

Wang further reduced the forwarding table by employing the compression scherne and considering both the distribution of prefix and the common prefix of prefixes belonged to the same segment. The size of the forwarding table is about
$430 \mathrm{~KB}$ and the number of memory accesses is between 1 and 3. The incremental update is not as easy as Huang's scheme [6], due to the same reason.

\section{The Proposed Scheme}

The proposed scheme is based on the indirect lookup mechanism, as shown in Fig. 1. The forwarding table is divided into the segment table (SEGT), the next hop / pointer table (NHPT) and the next hop table (NHT). The NHPT is replaced with the code word table (CWT) and the compressed NHPT(C-NHPT) to reduce the size of the forwarding table. Our scheme has two significant features. First, the CWT, the C-NHPT and the NHT are allocated in fixed size. The allocation in the manner of fixed size reduces the update overhead and the incremental update is feasible. Obviously, there are some disadvantages in the memory requirement and the number of memory accesses. We analyze these disadvantages in Section 4. Second, the logic delay of the lookup procedure in the hardware implementation is reduced using simple logics of smaller bits. For example, the logic delay of 8-bit adder is smaller than that of 16-bit adder.

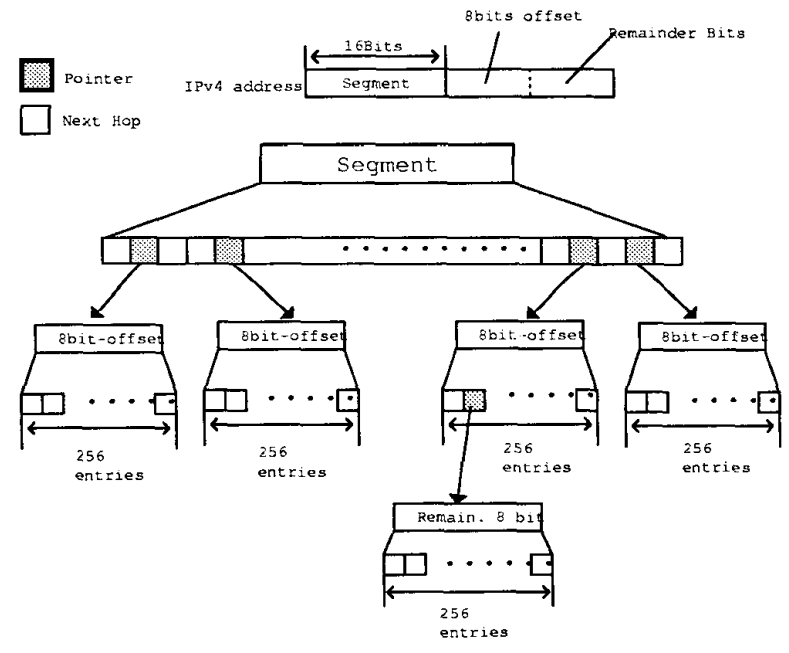

Figure 1. Indirect-lookup mechanism with fixed offset length

\subsection{Forwarding Table Structure}

The segment table stores all possible route prefixes that are not greater than 16 bits long. This table has $2^{16}$ entries, addressed from 0.0 to 255.255 . Since the length of the route prefix is variable, route prefixes shorter than 16-bits are expanded to prefixes 16 bits long, as in [9]. For example, 
$143.127 / 15$ is expanded to two route prefixes, $143.246 / 16$ and $143.247 / 16$. The expanded route prefix has the next hop of the original route prefix. Route prefixes longer than 16-bits are stored in the NHPT and the pointer information is recorded in the corresponding entry of the SEGT. So, the entry of the SEGT contains either the next hop, or the pointer to the NHPT entry. These information can be represented in 2 byte, so the entry is 2 byte wide

The NHPT contains all possible routes prefixes from 17 bits long to 24 bits long. Prefixes that share the first 16 bits, called a segment, are expanded to prefixes 24 bits long. Each segment is allocated 256 entiries in NHPT. The entry contains the same type of information as the entry in the SEGT entry and has the same width. The NHT contains all possible routes prefixes that are greater than 24 bits. Prefixes are expanded and allocated 256 entries in NHA. The entry contains the next hop and has 1 byte width.

Since most of prefixes are stored in NHPT, the key to reduce the table size is to compress NHPT. By employing the modified form of Degermark's compression scheme (called bitmap scheme), NHPT is replaced with the CWT and the C-NHPT. CWT contains 8-bits bitmap entries and 8-bits base entries to compute the index of C-NHPT. C-NHPT contains the compressed next hop / pointer. The original bitmap scheme reduces the table size significantly and increases the number of memory accesses by 2 , whereas our bitmap scheme reduces the table size less than the original, but increases the number of memory accesses by only 1 . Our bitmap scheme is similiar to Huang's scheme but we use 8-bit bitmap, so there are some gains in the logic delay.

We make the CWT and the C-NHPT using the sibling tree of the prefix range. The prefix can easily be converted to the prefix range as in [7]. The sibling tree is based on the fact that for any two distinct prefixes, either one is completely contained in the other, or the two prefixes have no entries in common. The CWT and the C-NHPT construction algorithm is shown in Fig. 2.

The sibling tree represents all prefixes in a segment. The example of the sibling tree is shown in Fig. 3. Each prefix is converted to the prefix range and is stored in the node of the sibling tree. The node also contains the pointer to its child and the pointer to its sibling. For example, $p_{1}=143.248 .0 / 17$ is converted to the prefix range $(0,127)$, the N1 has $p_{1}$ 's information, the pointer to $\mathrm{N} 3$, and the pointer to $\mathrm{N} 2$. The root node covers default-routes. The sibling tree is useful for updating the CWT and the C-NHPT.

While traversing the sibling tree in breadth-first, we obtain the range information, which is used to construct the CWT and the C-NHPT. In the example of Fig. 3, after visiting the root node, $\mathrm{R}$ is empty due to excluing the range of $\mathrm{N} 2$ and $\mathrm{N} 3$. After visiting $\mathrm{N} 2, \mathrm{R}=\left\{\left(0,23, p_{0}\right)\right.$, $\left.\left(32,79, p_{0}\right),\left(96,127, p_{0}\right)\right\}$ by excluding the range of $\mathrm{N} 3$ and N4. The rest of the node in $\mathrm{T}$ are processed by breadth-
The CWT and the C-NHPT construction algorithm

Input: The set $\mathrm{P}=\left\{p_{0}, p_{2}, p_{3}, \ldots, p_{n-1}\right\}$ of prefix of a segment, sorted by the prefix length.

Output: The CWT and C-NHPT of a segment

Step 1. Construct the sibling tree of the prefix range, $T$ for $P$. For any pairs of prefixes, $p_{i}$ and $p_{j}$, if $p_{i}$ contains $p_{j}$, then $p_{i}$ is a parent node of $p_{j}$.

Otherwise $p_{i}$ and $p_{j}$ is a sibling. The node, $n_{i}$, contains a range information, $\left(S_{i}, E_{i}, p_{i}\right)$

Step 2. Traverse the sibling tree $T$ in breadth-first search Let $R$ be a range information of a segment.

Mark the range of the visiting node in $R$, excluding a range of the children.

Step 3. Obtain a bitmap information from $R$.

Let CWT be a bitmap information of R, represented in 256 bits. For each $r_{i}$ in R, $S_{i}$ th bit of CWT is set to 1 and C-NHPT stores the next hop of $p_{i}$ corresponding to $r_{i}$. Step 4. Stop.

Figure 2. The CWT and the C-NHPT construction algorithm

first, and the procedure for constructing $\mathrm{R}$ is shown in Table

Prefixes:

$p 1=143.248 .0 / 17$

$\mathrm{p} 2=143.248 .128 / 17$

p3 $=143.248 .24 / 21$

$p 4=143.248 .80 / 20$

$\mathrm{p} 5=143.248 .208 / 20$

$p 6=143.248 .91 .77 / 27$

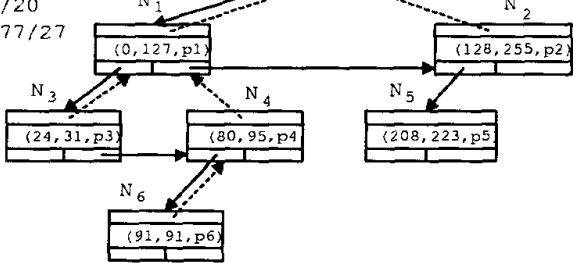

Figure 3. The example of the sibling tree

The CWT and the C-NHPT of a segment are obtained from R. In example of Fig. 3, the CWT has ten ones at bit $0,24,32,80,91,92,96,128,208$, and 224 and the rest of the CWT is marked to 0 . The C-NHPT is $\left\{p_{0}, p_{3}, p_{0}, p_{4}, p_{6}, p_{4}, p_{0}, p_{1}, p_{5}, p_{1}\right\}$

The time complexity of the proposed algorithm is $O\left(n^{2}\right)$, where $n$ denotes the number of prefixes in a segment, since the time complexity of the sibling tree construction is $O\left(n^{2}\right)$. The sibling tree has a good advantage in that it allows to update the CWT and the CNHPT partially. In example of Fig. 3, when $p_{7}=143.248 .224 / 24$ is inserted, only 
Table 1. The procedure for constructing $\mathbf{R}$

\begin{tabular}{|c|l|}
\hline visiting node & $\mathrm{R}$, the range information \\
\hline $\mathrm{N} 0$ & \\
\hline $\mathrm{N} 1$ & $\left(0,23, p_{0}\right),\left(32,79, p_{0}\right),\left(96,127, p_{0}\right)$ \\
\hline $\mathrm{N} 2$ & $\left(0,23, p_{0}\right),\left(32,79, p_{0}\right),\left(96,127, p_{0}\right)$, \\
& $\left(128,207, p_{1}\right),\left(224,255, p_{1}\right)$ \\
\hline N3 & $\left(0,23, p_{0}\right),\left(24,31, p_{3}\right),\left(32,79, p_{0}\right)$, \\
& $\left(96,127, p_{0}\right),\left(128,207, p_{1}\right)$, \\
& $\left(224,255, p_{1}\right)$ \\
\hline N4 & $\left(0,23, p_{0}\right),\left(24,31, p_{3}\right),\left(32,79, p_{0}\right)$, \\
& $\left(80,90, p_{4}\right),\left(92,95, p_{4}\right),\left(96,127, p_{0}\right)$, \\
& $\left(128,207, p_{1}\right),\left(224,255, p_{1}\right)$ \\
\hline N5 & $\left(0,23, p_{0}\right),\left(24,31, p_{3}\right),\left(32,79, p_{0}\right)$, \\
& $\left(80,90, p_{4}\right),\left(92,95, p_{4}\right),\left(96,127, p_{0}\right)$, \\
& $\left(128,207, p_{1}\right),\left(208,223, p_{5}\right)$, \\
& $\left(224,255, p_{1}\right)$ \\
\hline N6 & $\left(0,23, p_{0}\right),\left(24,31, p_{3}\right),\left(32,79, p_{0}\right)$, \\
& $\left(80,90, p_{4}\right),\left(91,91, p_{6}\right)\left(92,95, p_{4}\right)$, \\
& $\left(96,127, p_{0}\right),\left(128,207, p_{1}\right)$, \\
& $\left(208,223, p_{5}\right),\left(224,255, p_{1}\right)$ \\
\hline
\end{tabular}

the range of $\mathrm{N} 2$ is changed. We update the corresponding bit of CWT to N2 and insert $p_{7}$ to C-NHPT.

The CWT should be encoded as a sequence of bitmaps and bases with each 1 byte-width. The CWT contains 256bit streams and has been partition into a sequence of 8-bit streams. Then, theses streams are put into the bitmaps, sequentially. The base equals the number of ones accumulated in the bitmaps of previous entries. Figure 4 shows the partial CWT and the C-NHPT for prefixes in Fig. 3. For example, the first two entries of the CWT are $(10000000$, 0 ) and $(00000000,1)$, respectively. The base of the second entry has 1 since there is an one accumulated in the bitmaps of the previous entries.

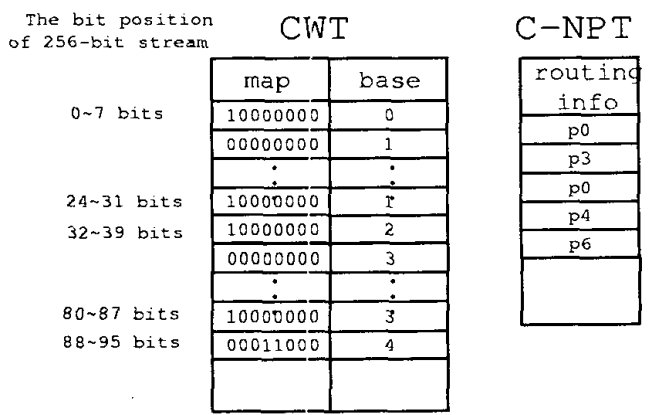

Figure 4. The example of the partial CWT and th C-NHPT
The search procedure consists of two steps. The first step is to find the entry of the CWT. The second step is to obtain the index to the C-NHPT by decoding the bitmap and the base. Let DA[m:n] be a bit subsequence of the destination address ranging from the $\mathrm{m}$-th bit to the $\mathrm{n}$-th bit. The first step is completed by indexing DA[16:20] which equals DA[16:23] DIV 8. In the second step, the index to the CNHPT is the sum of the base value and the number of ones from 0th bit to DA[21:23]th bit of the bitmap (DA[21:23] equals DA[16:23] MOD 8).

\subsection{Examples}

Consider the following examples of how lookups are performed on the table in Fig. 5. The forwarding table contains four route prefixes:143/8, 143.248/16, 143.248.24/24, 143.248.32.64/27. The first prefix requires entries from 143.0 to 143.255 except for 143.248 in SEGT. The second and the third prefixes are stored in the CWT and C-NHPT. The routing information of the fourth prefix is stored in NHT and the pointer to the NHT is contained in the CWT and the C-NHPT.

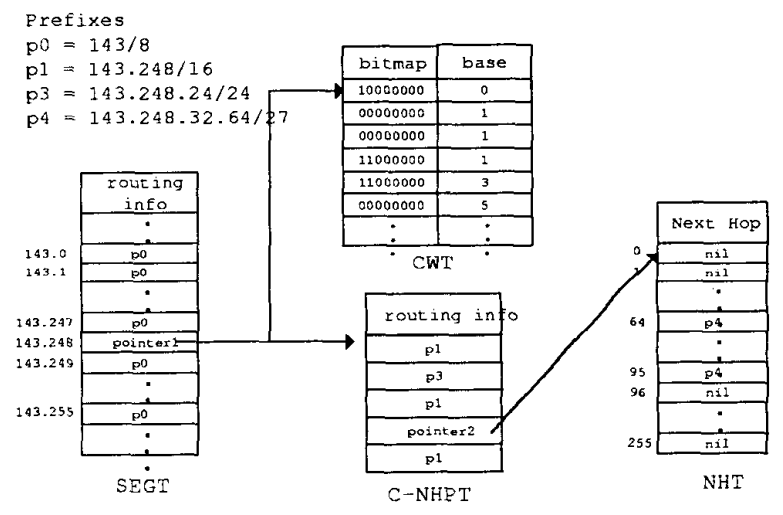

Figure 5. Example

Assume that the destination address of an incoming packet is 143.247.180.77. The first 16 bits are used as an index into SEGT and will return an entry with the next hop (p0). If the next packet arrives with the destination address 143.248.24.189, the first 16 bits are used as an index into SEGT, which indicates the CWT and the C-NHPT must be referenced. The pointer of the entry are concatenated with DA[16:20], and used as an index into the CWT. The fourth entry of the CWT is selected. The sum of the base (1) and the number of ones from 0 th bit to DA[21:23] th bit (1) is 2 . The pointer of the entry are concatenated with the sum, and used as an index into the C-NHPT. After three memory accesses, the table returns the next hop (p3). If 
the last packet arrives with the destination address with 143.248.32.70, then search the SEGT through the CWT. The decoding result in the CWT is 4 , and the fourth entry of the C-NHPT indicates that NHT must be referenced. $\mathrm{DA}[24: 31]$ is used as an index into NHT and will return the next hop (p4).

\subsection{Forwarding Table Updates}

Since each segment requires 64 bytes ( 2 byte $* 32$ entries) in the CWT, the memory can be partitioned into a sequence of 64-bytes block. If a prefix is inserted or deleted, only 64 bytes are updated. However, the problem is that the CWT and the C-NHPT share the memory address stored in SEGT, and the memory requirement of a segment in the C-NHPT is proportional to the number of prefixes. For example, if the memory requirement of a segment in the $C$ NHPT is 70 bytes, then two blocks ( 128 bytes) are allocated in both the CWT and the C-NHPT. That is, 64 bytes in the $\mathrm{CWT}$ are wasted. According to the simulation, most of segments require one block, so the memory waste is not large. The memory waste in a block is necessary for the memory management.

When a prefix is inserted, the size of the memory to rewrite is eight blocks of the C-NHPT and one block of the CWT in the worst case, and is 576 bytes. If the row size of SRAM is 2 byte and the access time of SRAM is 10ns, it takes about $3 \mu$ s to write the part of the memory. The average of the update time is faster, and the time to write one block of the CWT and the C-NHPT is merely 640ns.

\subsection{Hardware Implementation}

The high-level hardware architecture of the proposed scheme is shown in Fig. 6. The entry of the SEGT consists of two parts: the width flag (1bit) and the next hop / pointer (15bits). The width flag indicates whether the width of the entry in the C-NHPT is 1 byte or 2 byte. If the longest prefix in a segment is not greater than 24 bits, the C-NHPT stores the next hops which can be represented in 1 byte. Since there are few prefixes longer than 24 bits, the memory requirement of the C-NHPT is reduced.

DA $[0: 15]$ is used as an index of the SEGT. If the corresponding entry of the SEGT has a pointer, the memory address of the CWT entry is the value from 1st bit to 15 th bit of the SEGT entry * 32+ DA[16:23]. The base address of a segment in the CWT and the C-NHPT is the value from 1 st bit to 15 th bit of the SEGT entry * 32. Actually, the multiplication of $2^{n}$ does not need a multiplier. After the CWT entry is passed to the map filter and the $(8,4)$ counter, the offset address is obtained. If the width flag is 1 , the offset address is multiplied by 2 .

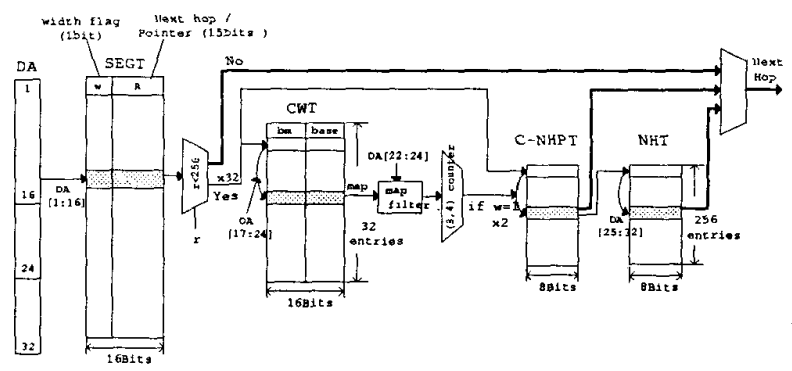

Figure 6. Example

\section{Performance Analysis}

The performance of the lookup scheme is evaluated by the delay of the table search, the memory requirement for the forwarding table, and the support for the incremental update of the forwarding table. The delay in the table search includes the delay for memory accesses and the logic delay. To evaluate the performance of the proposed scheme, we use the Internet-routing tables offered by the Internet Performance Measurement and Analysis(IMPA) Project [2]. The proposed algorithm is compared with two hardwareimplementable schemes:Huang's scheme [6] and DIR-24BASIC [5].

Table 2 shows the number of memory acceses and the average delay of the table search for three schemes. To obtain the logic delay, we investigate theses schemes in logic level and simulate the total delay in the table search. In our simulation, we use HD $0.65 \mu \mathrm{m}$ two-metal 3.3V CMOS technology and the gate delay is $0.21 \mathrm{~ns} .1,000,000$ IP addresses are randomly selected from the IP address space which the routing table convers, and simulated

Table 2. Lookup time comparison of three schemes

\begin{tabular}{|c|c|c|}
\hline Scheme & No. of memory accesses & avg delay \\
\hline Our scheme & $1-4$ & $17.3 \mathrm{~ns}$ \\
Huang's & $1-3$ & $19.5 \mathrm{~ns}$ \\
DIR-24-BASIC & $1-2$ & $50.2 \mathrm{~ns}$ \\
\hline
\end{tabular}

Since the proposed scheme uses the logic of smaller bits than Huang's and the total number of gates is smaller than Huang's, our scheme is slightly faster than Huang's. Whereas the delay of the whole logic in Huang's is $14.7 \mathrm{~ns}$, that of the proposed scheme is $7.8 \mathrm{~ns}$. In the worst case, total delays of the table search in Huang's, the propsed scheme, and DIR-24-BASIC are $44.7 \mathrm{~ns}, 47.8 \mathrm{~ns}$, and $100.2 \mathrm{~ns}$, re- 
spectively.

Table 3 shows the memory requirement and the support for the incremental update of three schemes. The forwarding table of Huang's scheme requires the smallest memory, but for the table update, it needs dual-port memory or dualmemory banks. This may degrade the performance due to the memory bandwidth contension. Our scheme requires more memory than Huang's scheme, but supports for the incremental table update.

\section{Table 3. The compiarion of three schemes in the memory requirement and the support for the incremental update}

\begin{tabular}{|c|c|c|}
\hline Scheme & $\begin{array}{c}\text { the memory } \\
\text { requirement }\end{array}$ & $\begin{array}{c}\text { the support for the } \\
\text { incremental update }\end{array}$ \\
\hline Our scheme & $470 \mathrm{~KB}-510 \mathrm{~KB}$ & Yes \\
Huang's scheme & $450 \mathrm{~KB}-470 \mathrm{~KB}$ & No \\
DIR-24-BASIC & $33 \mathrm{MB}$ & Yes \\
\hline
\end{tabular}

\section{Conclusion}

In this paper, we have proposed a fast and updatable lookup scheme that can be easily implemented in hardware. We have also presented the memory allocation policy that supports for the incremental update of the forwarding table. The memory allocation is performed in a block of fixed size, and the management of the memory is straightforward. The proposed CWT and C-NHPT construction algorithm is optimized for updating the table. In the prefix insertion and deletion, the partial update of the CWT and the C-NHPT is possible. It is remarkable that the forwarding table of our scheme is small enough to fit into SRAM, while supporting for the incremental update. We have investigated the recent hardware-implementable schemes in logic level and simulated the total clelay in lookup considering the logic delay. Since our lookup scheme can be implemented with the small-bit logic and 10ns SRAM, the average delay per one lookup is about $18 \mathrm{~ns}$. That is, our scheme can achieve $55.56 \times 10^{6}$ routing lookups/s in average.

\section{References}

[1] Network Wizards. Internet domain survey host count, http://www.nw.com.

[2] Internet Routing Table Statistics, http://www.merit.edu/ipma/routing_table/.
[3] M. Degermark, A. Brodnik, S. Carlsson, and S. Pink. Small Forwarding Tables for Fast Routing Lookups. Proceedings of the ACM SIGCOMM'97 conference on Applications, technologies, architectures, and protocols for computer communication, 27(4):3-14, Oct 1998.

[4] V. Fuller, T. Li, J.Yu, and K. Varadhan. Classless InterDomain Routing (CIDR): an Address Assignment and Aggregation Strategy. RFC 1519, Jan 1997.

[5] P. Gupta, S. Lin, and N. Mckeown. Routing Lookups in Hardware at Memory Access Speeds. Proceedings of IEEE INFOCOM' 98 Conference, pages 1240-1247.

[6] N.-F. Huang and S.-M. Zhao. Novel IP-Routing Lookup Scheme and Hardware Architecture for Multigigabit Switching Routers. IEEE Journal On Selected Areas in Communications, 17(6):1093-1104, Jun 1999.

[7] B. Lampson, V. Srinivasan, and G. Varghese. IP Lookups using Multiway and Multicolumn Search. IEEE/ACM Transactions on Networking, 7(3):324-334, Jun 1999.

[8] C.-T. C. Pi-Chung Wang and Y.-C. Chen. A Fast IP Routing Lookup Scheme. In Proceedings of the ICC 2000, 2:1140 $1144,2000$.

[9] V. Srinivasan and G. Varghese. Faster address Lookups using Controlled Prefix Expansion. ACM Transactions on Computer Systems, 17(1):1-40, Feb 1999.

[10] A. Tammel. How to survice as an ISP. Proceedings of Networld Interop'97, 1997. 